Comunicación

\title{
Canes Vagabundos en el Interior y Alrededores de Mercados Formales de Villa El Salvador, Lima, Perú
}

\author{
Stray Dogs inside and outside of Official Markets of Villa El Salvador \\ District in Lima, Peru
}

Carolina Chávez V. ${ }^{1,2}$, Néstor Falcón P. ${ }^{1,2,4}$, Daphne León ${ }^{1,2}$, Daniel Sánchez R. ${ }^{3}$

\section{Resumen}

\begin{abstract}
El objetivo del estudio fue estimar la población de canes vagabundos en el interior y exterior de 21 mercados formales del distrito de Villa el Salvador, Lima, Perú. Se elaboró una ficha epidemiológica y se registró el número de canes por mercado, características de los canes (sexo, tamaño, número de hembras lactantes, alteraciones de su condición física o salud) y su proximidad a puestos de expendio de alimentos. Se contabilizaron 518 y 568 canes en el interior y exterior de los mercados, respectivamente $(24.6 \pm 4.9$ y $27.1 \pm$ 7.7 canes por mercado, respectivamente). Los resultados muestran la necesidad de aplicar las normas correspondientes que regulan la presencia de canes vagabundos en los mercados a fin de prevenir potenciales problemas que pudieran afectar la salud pública.
\end{abstract}

Palabras clave: canes vagabundos, mercados, Villa el Salvador, salud pública

\section{Abstract}

The aim of the study was to estimate the population of stray dogs inside and outside 21 official markets of the district of Villa El Salvador, Lima, Peru. An epidemiological form was developed and the number and characteristics of dogs per market (sex, size, number of lactating females, welfare status), and their proximity to selling food stalls were registered. The total population of stray dogs was 518 and 568 inside and outside

\footnotetext{
${ }^{1}$ Facultad de Medicina Veterinaria y Zootecnia, Universidad Peruana Cayetano Heredia, Lima, Perú

${ }^{2}$ Grupo de Salud Pública Veterinaria (SAPUVET-PERÚ)

${ }^{3}$ Dirección de Salud Ambiental Lima Sur, Ministerio de Salud, Lima, Perú

${ }^{4}$ E-mail: nestor.falcon@upch.pe
}

Recibido: 14 de noviembre de 2014

Aceptado para publicación: 26 de octubre de 2015 
respectively ( $24.6 \pm 4.9$ y $27.1 \pm 7.7$ dogs per market respectively). The results showed the need to apply the relevant rules governing the presence of stray dogs in the markets to prevent potential problems that could affect public health.

Key words: dogs, markets, homeless, canine population

\section{INTRODUCCIÓN}

La tenencia poco responsable de animales por parte de sus propietarios en algunas localidades ha llevado a que la población de canes experimente un crecimiento casi exponencial (Butcher, 2000), lo cual se refleja en su desatención y abandono, y que ha conducido a la formación de poblaciones de canes considerados vagabundos o callejeros (Brusoni et al., 2007; Hernández et al., 2007).

Entre las causas de abandono de canes se encuentra el costo de mantenimiento, presencia de defectos físicos y disminución del afecto. Estas son las principales razones del crecimiento del número de canes vagos y no su reproducción durante su estado de abandono ya que allí tienen una escasa probabilidad de supervivencia (Álvarez y Domínguez, 2001; Guerra et al., 2007).

La Organización Mundial de Salud (OMS) estimó en 1992 una población canina mundial de 500 millones, $75 \%$ de los cuales fueron considerados como canes vagos (Butcher, 2000). En Valdivia, Chile, un estudio reveló que el $49 \%$ de una población de 5944 canes deambulaba libremente por las calles (Guttler, 2005); asimismo, otro estudio en San Martín de los Andes, Argentina, reveló que el $51 \%$ de una población de 5480 canes se criaban en forma independiente (Brusoni et al., 2007).

Los canes vagabundos representan un riesgo para las personas, por cuanto son potenciales transmisores de una gran cantidad de zoonosis (Guttler, 2005), entre ellas la toxocariasis, leptospirosis, giardiasis y ehrlichiosis (Dabanch, 2003), así como la rabia en regiones endémicas (Álvarez y Domínguez, 2001; ICAM, 2007). A ello se debe de sumar los accidentes por mordeduras, que pueden causar lesiones físicas, secuelas estéticas, infecciones y daño psicológico, además de representar un costo para la comunidad y el Estado (Glausiuss et al., 2000; AVMA, 2001).

Entre los perjuicios adicionales queocasionan los canes vagabundos se encuentra la contaminación ambiental debido a los destrozos de las bolsas de basura en busca de alimento (Álvarez y Domínguez, 2001) y las heces en calles y parques públicos, los cuales representan focos de contaminación y transmisión de enfermedades (Andresiuk et al., 2004; Milano y Oscherov, 2005).

Los canes vagabundos tienden a concentrarse en los mercados de abasto, especialmente en barrios populares debido al deficiente control sanitario y acúmulo de basura en sus alrededores, donde los canes encuentran su alimento (Álvarez y Domínguez, 2001; Ibarra et al., 2006). La presencia de estos animales en el interior y exteriores de mercados en ciudades del Perú se mantiene pese a lo dispuesto en el Art. 34 del Reglamento Sanitario de Funcionamiento de Mercados de Abasto (2003) que prohíbe la presencia de mascotas en el interior de los mercados y la Ley que Regula el Régimen Jurídico de Canes que prohíbe la permanencia de estos animales sin sujeción en lugares públicos (Ley 27596, 2002).

En los mercados del distrito de Villa el Salvador, Lima, Perú, se presentaba un creciente número de canes vagabundos, de allí que el objetivo del presente estudio fue esti- 
mar la cantidad y características de los canes que deambulan en los mercados del distrito.

\section{Materiales y Métodos}

El estudio se realizó en el distrito de Villa el Salvador, ubicado en la costa central de Lima. El distrito tiene una extensión de 35546 $\mathrm{km}^{2}$ y una población de 388588 habitantes, y cuenta con 41 mercados formales.

El estudio correspondió a una investigación de tipo transversal y descriptivo. Se seleccionó el $50 \%$ de los mercados formales, excluyendo aquellos que eran de tamaño pequeño (menos de 80 puestos) y los que no se encontraban en funcionamiento. En cada mercado, la población objetivo fueron los canes vagabundos (considerados como aquellos que en ese momento no se encuentran bajo control directo refrenado por una cadena u otro medio de sujeción, lo que incluye a canes con y sin dueño) encontrados en el interior y exterior de dichos mercados. La recolección de los datos se realizó entre los meses de noviembre y diciembre de 2012 y contó con la autorización de la Asociación de Mercados de Villa el Salvador (FUCOMIVES).

Para estimar la población de canes se elaboró una ficha epidemiológica, donde se registró el número de animales por mercado, sexo (macho, hembra o no determinado), tamaño (pequeño $[<10 \mathrm{~kg}$ ], mediano [10 a 15 $\mathrm{kg}]$ o grande [ $>15 \mathrm{~kg}]$ ) y proximidad del can a puestos de expendio de alimentos (carnes, abarrotes, comida preparada, verduras o frutas).

La observación y conteo de canes en el interior de los mercados se realizó entre las 08:00 y 11:00 horas, y en los alrededores o afueras de los mercados entre las 14:00 y 17:00 horas. En cada sesión se recorrió dos mercados por día y una hora por mercado en horario matutino y una hora en horario de la tarde.
Para el recorrido en el interior del mercado se extrapoló la metodología descrita por la WSPA (2007) en la publicación «Censando poblaciones de perros deambulantes: guía metodológica» realizando un paralelo entre calles y pasillos. Se recorrió el total de pasillos evitando repetirlos, registrando el número de canes vagabundos y sus características según la ficha epidemiológica. Para evitar contar un mismo animal en más de una oportunidad, se registró alguna característica particular para diferenciarlo. En casos donde la cantidad de canes era grande se hizo un registro fotográfico para su posterior registro. El recorrido en el exterior del mercado se hizo entre dos a cuatro lados del mercado, dependiendo si colindaban con otros comercios o viviendas. El recuento de canes vagabundos se realizó en dos ocasiones en cada mercado.

Para el análisis de datos se obtuvo el promedio de canes contabilizados en cada visita. Se calculó el total, media aritmética, desviación estándar y valores extremos para la cantidad de canes vagabundos dentro y fuera de los mercados. Asimismo, se determinó la frecuencia de canes vagabundos para cada variable del estudio

\section{Resultados}

El número de canes vagabundos registrado en los 21 mercados formales de Villa El Salvador fue de 518 en el interior y 568 en el exterior. El promedio de canes por mercado fue de 24.6 y 27.1 en cada caso (Cuadro 1).

En el interior de los mercados predominaron los canes machos pero no se pudo precisar el sexo en la mayoría de los canes que se encontraban en los exteriores de los mercados. Por otro lado, la mayor parte de los canes en los interiores y exteriores de los mercados fueron de tamaño mediano y grande (Cuadro 2). 
Cuadro 1. Presencia de canes vagabundos en 21 mercados de Villa el Salvador (Lima, Perú, 2012)

\begin{tabular}{ccccc}
\hline \multirow{2}{*}{ Área del mercado } & \multirow{2}{*}{ Total de canes } & Canes por mercado & \multicolumn{2}{c}{ Valores extremos } \\
\cline { 3 - 5 } & & Promedio \pm d.e. & Mínimo & Máximo \\
\hline Interna & 518 & $24.6 \pm 11.5$ & 9 & 56 \\
Externa & 568 & $27.1 \pm 18.1$ & 11 & 79 \\
\hline
\end{tabular}

Cuadro 2. Características de canes vagabundos en 21 mercados del distrito de Villa el Salvador (Lima, Perú, 2012)

\begin{tabular}{llcccc}
\hline \multirow{2}{*}{ Variable } & & \multicolumn{2}{c}{$\begin{array}{c}\text { Interior del mercado } \\
(\mathrm{n}=518)\end{array}$} & \multicolumn{2}{c}{$\begin{array}{c}\text { Exterior del mercado } \\
(\mathrm{n}=568)\end{array}$} \\
\cline { 3 - 6 } & & $\mathrm{N} .^{\circ}$ & $\%$ & $\mathrm{~N} .^{\circ}$ & $\%$ \\
\hline \multirow{2}{*}{ Sexo } & Macho & 231.5 & 44.7 & 119.0 & 21.0 \\
& Hembra & 85.5 & 16.5 & 66.5 & 11.7 \\
& No determinado & 201.0 & 38.8 & 382.5 & 67.3 \\
\multirow{2}{*}{ Tamaño } & Pequeño & 124.0 & 23.9 & 89.0 & 15.7 \\
& Mediano & 206.0 & 39.8 & 237.5 & 41.8 \\
& Grande & 188.0 & 36.3 & 241.5 & 42.5 \\
\hline
\end{tabular}

Entre los canes registrados en el interior del mercado, el $37.5 \%$ se observó cerca de los puestos de comercialización de carnes, $25.7 \%$ en los puestos de abarrotes, $23.2 \%$ en los puestos de comida, $9.5 \%$ en los puestos de verdura y la diferencia (4.1\%) en los puestos de frutas.

\section{Discusión}

La cantidad de canes vagabundos encontrados tanto dentro como fuera de 21 mercados del distrito de Villa el Salvador, Lima, refleja el incumplimiento del Reglamento Sanitario de Funcionamiento de Mercados de Abasto y la Ley que Regula el Régimen Jurídico de Canes. El primero, establece que se encuentra prohibido el ingreso de canes a los mercados con la finalidad de asegurar la calidad sanitaria de los alimentos y bebidas; y el segundo, menciona que los canes no pueden permanecer en ningún lugar público sin sujeción, a menos que estos formen parte de un servicio de vigilancia formal.

La presencia de canes vagabundos se debería mayormente a la tenencia irresponsable de numerosos propietarios que no ejercen un control adecuado de sus animales, permitiendo su permanencia en las calles sin sujeción alguna. Álvarez y Domínguez (2001) e Ibarra et al. (2006) mencionan que los canes vagabundos se dirigen a los mercados debido a que en estos lugares encuentran alimento que lo obtienen en puestos de alimen- 
to o en los acúmulos de basura en los alrededores. Sin embargo, no se puede descartar que existan animales que acompañen a sus propietarios (compradores o dueños de puestos), lo que explicaría la presencia de canes en puestos en lo que no necesariamente accederían a alimento (ej. abarrotes). La presencia de canes vagabundos se debería además a que no existe ninguna barrera que limite su acceso al interior de los mercados (barreras físicas o seguridad) y menos aún hacia los lugares donde se apila la basura en el exterior de los mismos.

La diferenciación del sexo de los animales fue mayor en el interior del mercado debido a la facilidad de observarlos de cerca entre los puestos de expendio. Sin embargo, la confirmación del sexo de las hembras fue más difícil cuando el animal se encontraba en posición dorsal respecto a la mirada del evaluador, así como la presencia de pelo largo que bloqueaba la visión. En caso de los canes que se encontraban en el exterior, la diferenciación del sexo se dificultaba, además, por la mayor distancia entre los canes y el observador y por una mayor aglomeración de los canes. Sin embargo, entre los animales que se logró diferenciar el sexo predominaron los canes machos. Estos hallazgos fueron similares a los encontrados por Ochoa $e t$ $a l$. (2014) en un estudio de perros vagabundos en el distrito de Los Olivos, en donde se esgrime como hipótesis que la presencia de perros machos estaría asociada a la predilección por una mayor tenencia de este tipo de animales a fin de evitar las molestias de preñez no deseadas entre sus mascotas y que ello se reflejaría también en los perros vagabundos quienes se encontrarían en la calle por el abandono a los que fueron sometidos por quienes fueran sus propietarios anteriormente.

Los tamaños que predominaron fueron los medianos (aproximadamente entre 10 a $15 \mathrm{~kg})$ y grandes $(>15 \mathrm{~kg})$. Este tipo de animales son generalmente aceptados para cumplir funciones de vigilancia, a diferencia de los animales pequeños que son mantenidos como animales falderos, por lo que sus dueños (de tenerlos) tienen mayor cuidado de dejarlos libres debido al temor de que sean agredidos por animales de mayor tamaño o puedan sufrir algún accidente de tránsito. La importancia del tamaño en los animales vagabundos radica en la capacidad de procrear un mayor número de crías, lo que influiría en el rápido crecimiento de la población canina vagabunda debido a la falta de control en la reproducción.

La mayor cantidad de animales se encontró en puestos de venta de carnes. En estos lugares suele existir restos y despojos de carnes y vísceras que los trabajadores les ofrecen a los canes. Esta práctica es riesgosa debido a que existen enfermedades que pueden ser transmitidas por esta vía como la equinococosis, la cual se adquiere por el consumo de la forma larvaria del Echinococcus granulosus, el quiste hidatídico que se encuentra en las vísceras de animales como bovinos y ovinos, entre otros. Otro tipo de puesto a los que se dirigían los canes en busca de comida son aquellos que expenden alimentos al público. En estos lugares los animales también tienen acceso a restos utilizados para su alimentación por parte de los dueños de los puestos y de los clientes.

Considerando de que no deben de haber canes vagabundos dentro ni fuera de los mercados y que ellos está normado debidamente por el Reglamento Sanitario de Funcionamiento de Mercados de Abasto y la Ley que Regula el Régimen Jurídico de Canes, los resultados del estudio deben de ser observados por las autoridades municipales y a partir de ella realizar las acciones de intervención que le faculta las normas (especialmente la Ley 27596). Asimismo, se debe de seguir difundiendo las campañas de sensibilización y educación acerca de la tenencia responsable de animales de compañía y campañas de control poblacional de canes, donde en este último punto se debe de incluir a los canes vagabundos sin dueño. 


\section{Conclusiones}

La presencia de canes en los mercados y alrededores muestra el incumplimiento del Reglamento Sanitario de Funcionamiento de Mercados de Abasto y la Ley que Regula el Régimen Jurídico de Canes.

\section{Literatura Citada}

1. Andresiuk M, Rodriguez, F, Denegri M, Sardella N, Hollmann P. 2004. Relevamiento de parásitos zoonóticos en materia fecal canina y su importancia para la salud de los niños. Arch Argent Pediatr 102: 325-329.

2. Álvarez E, Domínguez J. 2001. Programa para el control integral de la población canina. Rev AMMVEPE 12(3): 83-91.

3. [AVMA]American Veterinary Medical Association. 2001. A community approach to dog bite prevention. J Am Vet Med Assoc 218: 1732-1749.

4. Brusoni C, Dezzotti A, Fernández J, Lara J. 2007. Tamaño y estructura de la población canina en San Martín de los Andes (Neuquén). Analecta Vet 27(1): 11-23.

5. Butcher R. 2000. La implementación de programas de control de animales vagos, los efectos de las diferencias económicas y culturales. MEVEPA 14(3): 40-46.

6. Dabanch J. 2003. Zoonosis. Rev Chil Infectol 20(Sup1 1): S47-S51. doi: 10.4067/S0716-10182003020100008

7. Glausiuss G Ascione I, Sehabiague G. 2000. Mordeduras por animales en la edad pediátrica. Arch Pediatr Urug 71: 24-30.

8. Guerra Y, Echagarrúa Y, Marín E, Mencho J, Marín A, Pascual T, et al. 2007. Factores que conllevan al abandono de perros en una región de Cuba. REDVET 8(12). [Internet]. Disponible en: http://www.veterinaria.org/revistas/ redvet/n121207.html\#120704

9. Guttler V. 2005. Análisis de algunas características de la población canina relacionadas con mordeduras e hidatidosis humana en la provincia de Valdivia. Tesis de Médico Veterinario. Chile: Univ Austral de Chile. 64 p.

10. Hernández $R$, Núñez F, Pelayo L. 2007. Potencial zoonótico de las infecciones por helmintos intestinales en perros callejeros de Ciudad de La Habana. Revista Cubana de Medicina Tropical. 59(3). [Internet]. Disponible en: http:/ $/$ scielo.sld.cu/scielo.php?script $=$ sci_arttext \& pid = S 0375 07602007000300009\&lng=es

11. Ibarra L, Espínola F, Echevarría M. 2006. Factores relacionados con la presencia de perros en las calles de la ciudad de Santiago. Avances Cienc Vet 21(1-2): 21-26. doi: 10.5354/07195273.2006 .1384

12. [ICAM] Coalición Internacional para el Manejo de Animales de Compañía. 2007. Guía para el manejo humanitario de poblaciones caninas. [Internet]. Disponible en: http://www.icam-coalition. org/downloads/Guia_Para_El_ Manejo_Humanitario_de_Poblaciones_ Caninas_Spanish.pdf

13. Ley $N .^{\circ} 27596$ Régimen jurídico de canes. El Peruano 2002. p 225240225244.

14. Milano A, Oscherov E. 2005. Contaminación de aceras con enteroparásitos caninos en Corrientes, Argentina. Parasitol Latinoam 60: 82-85.

15. Ochoa Y, Falcón N, Zuazo R, Guevara P. 2014. Estimación de la población de perros callejeros en el distrito de Los Olivos, Lima, Perú. Rev Inv Vet Perú 25: 366-373. doi:10.15381/rivep. v25i3. 10114

16. Reglamento Sanitario de Funcionamiento de Mercados de Abasto. 2003. Resolución Ministerial N ${ }^{\circ}$ 282-2003-SADM. El Peruano. p 246762. 
17. [WSPA] World Society for the Protection of Animals. 2007. Censando poblaciones de perros deambulantes: guía metodológica [Internet]. Disponible en: http://xa.yimg.com/kq/groups/ 15296103/27554350/name/resources_ Companion\% 2520Animals_ true Surveying- roaming-dog-popula-tionsguidelines-on-methodologySpanish_tcm35-8158.pdf 